\title{
Defective Infection of Bovine Herpesvirus 1 in Non-Permissive Murine Cells
}

\author{
Kazuo NAKAMICHI ${ }^{1)}$, Yasunobu MATSUMOTO ${ }^{1)}$ and Haruki OTSUKA ${ }^{1) *}$ \\ ${ }^{1)}$ Department of Global Animal Resource Science, Graduate School of Agricultural and Life Sciences, The University of Tokyo, 1-1-1 \\ Yayoi, Bunkyo-ku, Tokyo 113-8657, Japan
}

(Received 27 March 2001/Accepted 25 May 2001)

ABSTRACT. The defective growth of bovine herpesvirus 1 (BHV-1) was analyzed in non-permissive murine embryo fibroblast, BALB/3T3 A31-1-1 (A31) cells. BHV-1 was able to attach and penetrate into A31 cells at similar levels that were seen in semi-permissive cells. Once penetrated into A31 cells, BHV-1 was efficiently transported to nuclei, but the onset of expression of immediate early (IE) protein and viral DNA replication was not observed. These data suggest that the viral replication of BHV-1 in A31 cells is arrested at the point prior to the expression of IE proteins.

KEY WORDS: BHV-1, growth defect.

J. Vet. Med. Sci. 63(10): 1139-1142, 2001

Bovine herpesvirus 1 (BHV-1) is a member of alphaherpesvirus group and causes various diseases such as infectious bovine rhinotracheitis, abortions, conjunctivitis, and infectious pustular vulvovaginitis in cattle [1]. BHV-1 has a relatively narrow host cell range in vivo and in vitro when compared to other alphaherpesviruses such as herpes simplex virus (HSV) and pseudorabies virus (PrV). BHV-1 exhibits productive replication in bovine-derived cell lines such as Madin-Darby bovine kidney (MDBK) cells, while viral replication is impaired in cell lines derived from diverse vertebrate species. Replication of BHV-1 is strictly restricted and no infectious progeny is produced in cell lines such as murine embryo fibroblast, BALB/3T3 A31-1-1 (A31) cells [5]. On the other hand, BHV-1 exhibits limited replication in hamster lung-derived $\mathrm{HmLu}-1$ cells [4]. Hence, A31 cells are non-permissive and HmLu-1 cells are semi-permissive for BHV-1.

It has been reported that the host cell range of HSV-1 [2, 9, 12, 13], PrV [8, 10] and canine herpesvirus (CHV) [7] is determined at the level of penetration due to the specificity of cellular receptor (s). However, in the case of the abortive infection of murine resident peritoneal macrophage by HSV-1, Morahan et al. demonstrated that the block in HSV1 multiplication localized at the level of expression of the early to delayed-early viral genes [3].

In our previous study, we have reported that attachment and penetration of $\mathrm{BHV}-1$ were significantly reduced in semi-permissive HmLu-1 cells compared to those in permissive MDBK cells [4]. We also analyzed the attachment and penetration properties of a recombinant BHV-1, designated as BHV-1/TF7-6, expressing PrV glycoprotein B $(\mathrm{PgB})$ and glycoprotein $\mathrm{C}(\mathrm{PgC})[5]$ and found that the defective entry of BHV-1 into semi-permissive HmLu-1 and non-permissive A31 cells was greatly improved by the expression of $\mathrm{PgB}$ and $\mathrm{PgC}$ [5]. In this study, we have ana-

\footnotetext{
* Correspondence to: Otsuka, H., Department of Global Animal Resource Science, Graduate School of Agricultural and Life Sciences, The University of Tokyo, Yayoi 1-1-1, Bunkyo-ku, Tokyo 113-8657, Japan.
}

lyzed the bottlenecks of BHV-1 growth in non-permissive A31 cells in more details.

At first, we compared the attachment and penetration rates of BHV-1 to A31 cells with those to MDBK and HmLu-1 cells according to the method described previously [4-7]. Figure 1 A shows the attachment of BHV-1 to MDBK, HmLu-1 and A31 cells. Cell monolayers in 6-well culture plates were incubated with wild-type BHV-1 (LA strain) at a multiplicity of infection (MOI) of 10 (10 PFU/ cell) for $2 \mathrm{hr}$ at $4^{\circ} \mathrm{C}$, scraped and washed with PBS, and total (cellular and viral) DNA was extracted. The amount of BHV-1 DNA was measured by quantitative competitive polymerase chain reaction (QCPCR) assay [4-7]. BHV-1 attached to cells at $4{ }^{\circ} \mathrm{C}$ but did not penetrate into cells. The amounts of BHV-1 attached to HmLu-1 or A31 cells were at a similar level but was lower by approximately $50 \%$ when compared to those to MDBK cells. When temperature was shifted to $37^{\circ} \mathrm{C}$, attached $\mathrm{BHV}-1$ penetrated into cells and became resistant to trypsin treatment. In Fig. $1 \mathrm{~B}$, the amount of penetrated BHV-1 into cells was shown as the percentage of trypsin resistant BHV-1 over attached one. Cells were incubated with BHV-1 at an MOI of 10 for $2 \mathrm{hr}$ at $4{ }^{\circ} \mathrm{C}$, washed with PBS and overlaid with prewarmed medium containing an inhibitor of herpesvirus DNA replication, phosphonoacetic acid (PAA, $400 \mu \mathrm{g} / \mathrm{ml}$ ). At 0,2 and $5 \mathrm{hr}$ after temperature shift, extracellular viruses were removed by trypsin treatment, and the amount of viral genome was measured by QCPCR assay. Penetration of BHV-1 into A31 or HmLu-1 cells was significantly reduced when compared to that into MDBK cells. At $5 \mathrm{hr}$ postinfection (p.i.), approximately $20 \%$ of attached BHV-1 penetrated into HmLu-1 or A31 cells, while about $80 \%$ of BHV1 could penetrate into MDBK cells. From these results, it is apparent that the attachment and penetration of BHV-1 were significantly impaired in non-permissive A31 cells when compared to those in permissive MDBK cells. However, the attachment and penetration in A31 cells were similar levels that were seen in semi-permissive HmLu-1 cells, suggesting that the virus attachment and penetration do not 
totally determine the non-permissiveness of A31 cells to BHV-1 infection.

Following attachment and penetration processes, nucleocapsid and tegument of BHV-1 are transported to the nucleus of target cells [14]. In the experiment shown in Fig. $2 \mathrm{~A}$, nuclear transport of BHV-1 penetrated into MDBK, HmLu-1 and A31 cells was examined. Cells were infected with BHV-1 in the same procedure as penetration assay, and the nuclear fraction was extracted as described previously [16]. BHV-1 DNA in each sample was quantified by QCPCR method. In each cell type, the amount of BHV-1 transported to the cell nuclei steeply increased between 0 and $2 \mathrm{hr}$ and went on increasing gradually between 2 and 8 $\mathrm{hr}$ after virus penetration. The intracellular transport of BHV-1 to cell nuclei in A31 cells was as efficient as that in MDBK and HmLu-1 cells. These data suggested that the restriction of BHV-1 growth in A31 cells did not occur at the step between virus entry and nuclear transportation of BHV-1 DNA.

In order to examine growth defect of BHV-1 posterior to nuclear transport, we monitored the replication of BHV-1 genome in A31 and HmLu-1 cells by using QCPCR method. Cells in 12-well culture dishes were incubated with wildtype BHV-1 or BHV-1/TF7-6 at an MOI of 10 . After an additional incubation for $2 \mathrm{hr}$ at $37^{\circ} \mathrm{C}$, remaining extracellular viruses were inactivated by low-pH treatment [6], and the cells were overlaid with culture medium. Immediately thereafter $(0 \mathrm{hr})$ and at 6,12 and $24 \mathrm{hr}$ p.i., cells and supernatants were harvested, and total DNA was extracted by using DNAZOL reagent (Gibco BRL) as described previously [6]. The amount of viral DNA in each sample was quantified by QCPCR (Fig. 2B). In semi-permissive HmLu- 1 cells, the viral DNA of both BHV-1 strains was multiplied gradually between 6 and $24 \mathrm{hr}$ p.i. For BHV-1/ TF7-6 strain, which expresses $\mathrm{PgB}$ and $\mathrm{PgC}$, the amount of viral DNA was markedly higher than that of wild-type BHV-1. This would suggest that the increased viral attachment and penetration into $\mathrm{HmLu}-1$ cells resulted in increased DNA replication of BHV-1. In contrast, no increase in viral DNA of wild-type BHV-1 or BHV-1/TF76 was observed in A31 cells. Therefore, increased entry of BHV-1/TF7-6 in A31 cells did not result in increase in viral DNA replication.

To examine the restriction of viral growth after nuclear transport of BHV-1 in more detail, we compared the expression of BHV-1 infected cell-specific protein 4 (BICP4) in HmLu-1 and A31 cells by indirect immunofluorescence assay. BICP4, which is classified as immediate early (IE) protein encoded by BHV-1, acts as a regulator of other classes (early, late) of viral genes [11]. Indirect immunofluorescence assay was performed essentially as described previously [15]. HmLu-1 and A31 cells were mock infected or infected with BHV-1 (LA) and BHV-1/TF7-6 at an MOI of 10 for $1 \mathrm{hr}$ at $37^{\circ} \mathrm{C}$, washed and overlaid with culture medium. At $24 \mathrm{hr}$ p.i., cell were fixed with methanol and reacted with mouse anti-BICP4 antiserum [4]. Then the cells were stained with fluorescein isothiocyanate (FITC)-
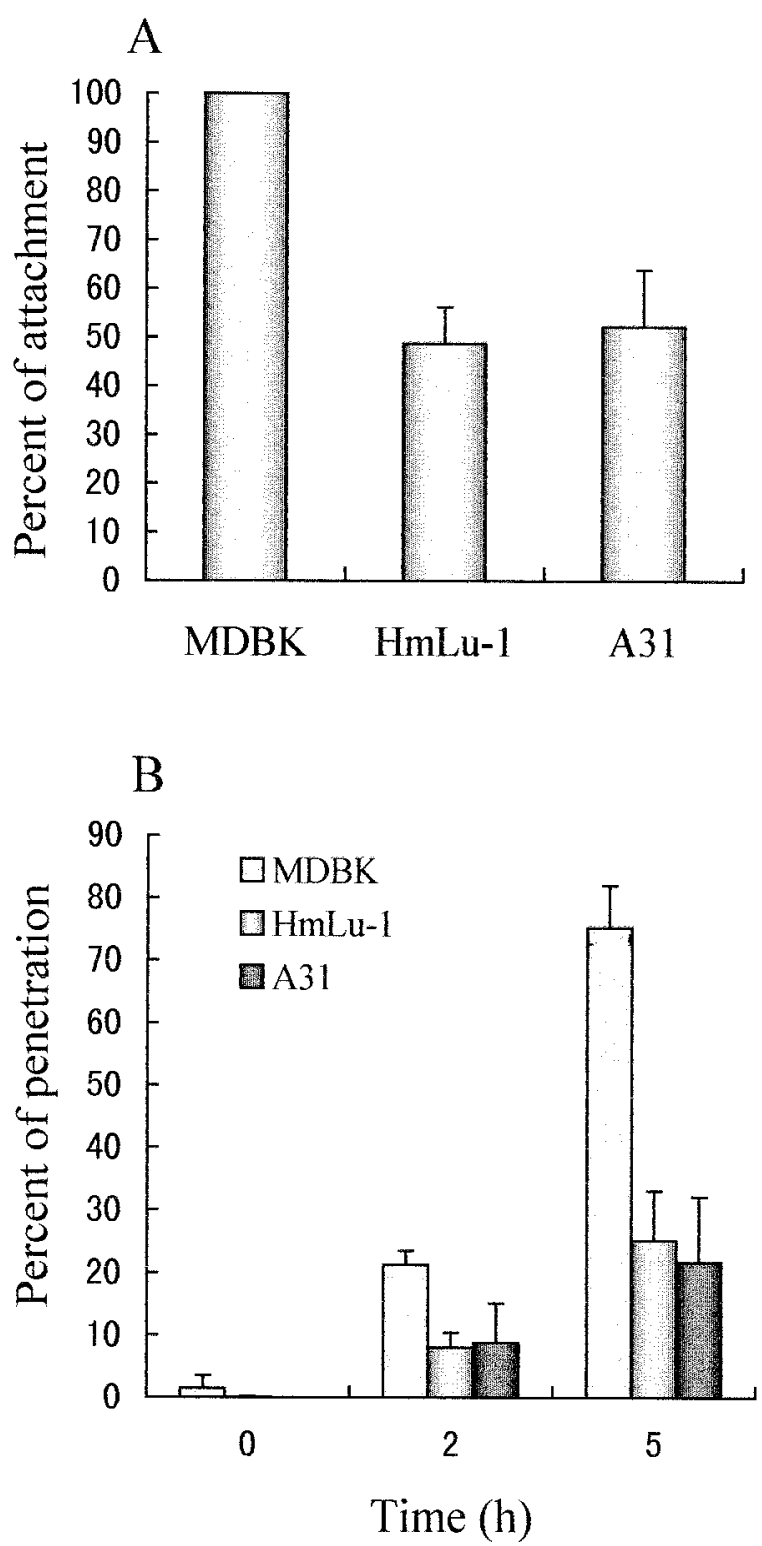

Fig. 1. Attachment of BHV-1 to cells (panel A). Cell monolayers were incubated with wild-type BHV-1 (LA) at an MOI of 10 for $2 \mathrm{hr}$ at $4^{\circ} \mathrm{C}$, and total DNA was extracted. The amount of BHV-1 DNA was measured by QCPCR assay. Relative copy number of attached BHV-1 compared to MDBK cells is indicated. Penetration of BHV-1 into cells (panel B). Cells were incubated with BHV-1 (LA) at an MOI of 10 for $2 \mathrm{hr}$ at $4^{\circ} \mathrm{C}$, washed and overlaid with prewarmed medium containing PAA. At 0,2 and $5 \mathrm{hr}$ after temperature shift, extracellular viruses were removed by trypsin treatment, and total DNA was extracted. The amount of viral DNA was measured by QCPCR assay. The percentage of genome copies of BHV-1 surviving the trypsin treatment was calculated with reference to the values for virus attachment for $2 \mathrm{hr}$ at $4^{\circ} \mathrm{C}$ and is shown as percent penetration. For each panel, values represent averages of triplicate samples, while the vertical lines indicate the standard deviation. 

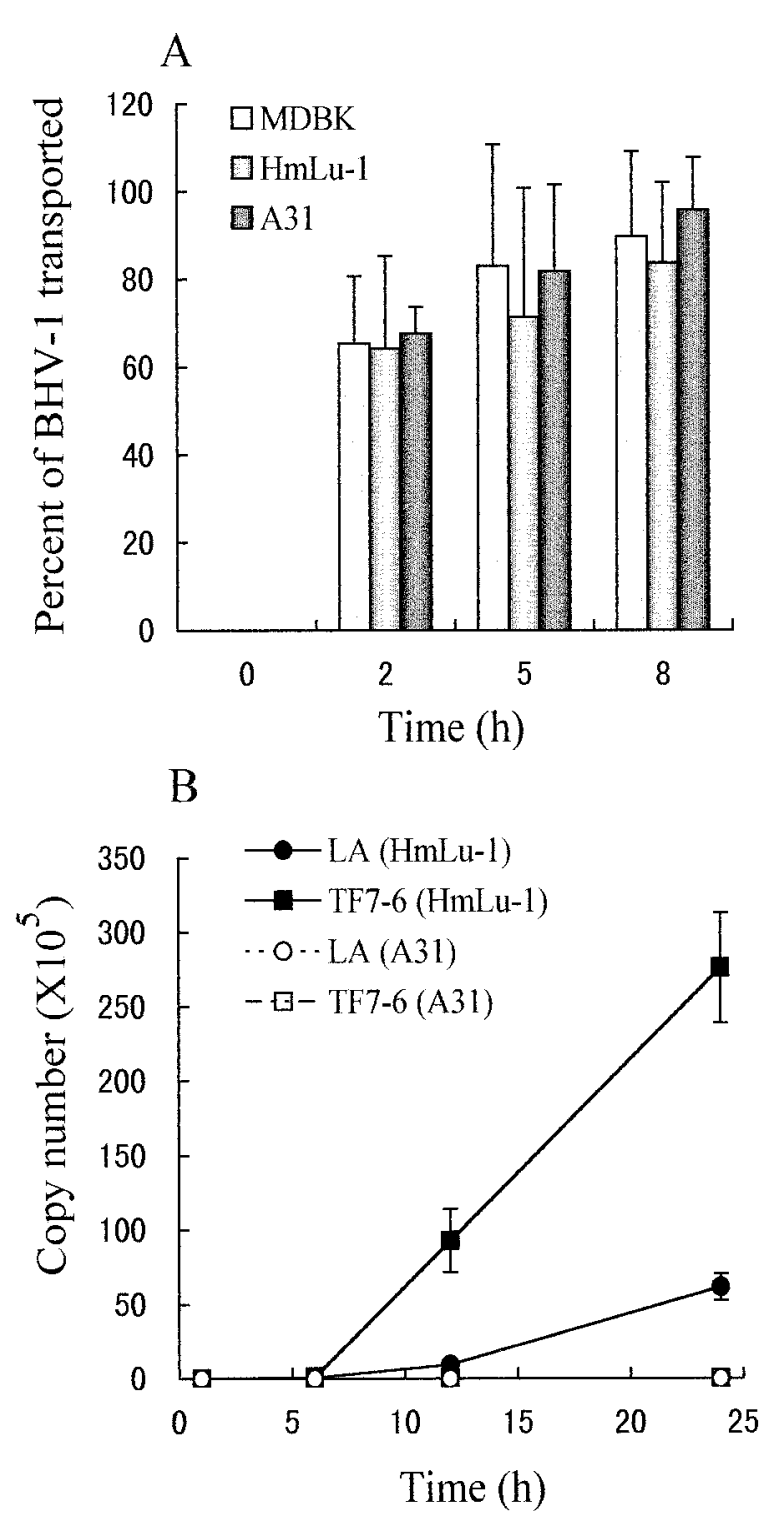

Fig. 2. Intracellular transport of input BHV-1 to cell nuclei (panel A). Cells were infected with BHV-1 (LA) at an MOI of 10 for 2 hr at $4^{\circ} \mathrm{C}$, washed and overlaid with prewarmed medium containing PAA. At $0,2,5$ and $8 \mathrm{hr}$ after temperature shift, DNA was extracted from nuclear fractions of infected cells, and the amount of viral DNA was measured by QCPCR assay. The percentage of genome copies of BHV-1 in nuclear fraction was calculated with reference to the values for viral penetration at the same time points and is shown as percent transportation. DNA replication of BHV-1 (panel B). Cells were infected with BHV1 (LA) and BHV-1/TF7-6 at an MOI of 10, and cells and supernatants were harvested at the time points indicated. Total DNA was extracted, and the amount of viral DNA was measured by QCPCR assay. For each panel, values represent averages of triplicate samples, while the vertical lines indicate the standard deviation. conjugated goat anti-mouse IgG antibody and examined under a fluorescent microscope (Fig. 3). When semi-permissive HmLu-1 cells were infected with the wild-type BHV-1 strain, the expression of BICP4 was detected as early as early as $3 \mathrm{hr}$ p. i., and the signals got stronger at later time points (data not shown). For BHV-1/TF7-6, the expression of BICP4 in $\mathrm{HmLu}-1$ cells was significantly greater than that for wild-type BHV-1 at $24 \mathrm{hr}$ p.i. (Fig. 3, upper panels). This agrees with the enhanced entry of BHV1 by the expression of $\mathrm{PgC}$ and $\mathrm{PgB}$. In contrast, no signal was observed in non-permissive A31 cells for either wildtype BHV-1 or BHV-1/TF7-6 at 24 hr p.i. (Fig. 3, lower panels).

The results of current study can be summarized as follows: 1) Virus attachment and penetration of BHV-1 were impaired in A31 cells at similar levels that were seen in semi-permissive HmLu-1 cells (Fig. 1). 2) Intracellular transport of BHV-1 to cell nuclei was not arrested (Fig. 2A). 3) Expression of IE product, BICP4, and the onset of DNA replication were not observed in A31 cells (Fig. 2B and Fig. 3). 4) The increase in virus entry of BHV-1/TF7-6 did not induce either the expression of BICP4 or the replication of viral DNA. Results obtained here clearly suggest that the BHV-1 replication in A31 cells was completely arrested at point(s) posterior to nuclear transport and prior to expression of IE protein that regulates other classes of viral genes. At this time, the molecular mechanism of this blockage in A31 cells remains to be identified. We hypothesize that the BHV-1 IE promoter activity is impaired in non-permissive A31 cells. It is also possible that the release of BHV-1 genome from nucleocapsid into cell nuclei may be abortive in non-permissive A31 cells.

ACKNOWLEDGMENTS. This work was partly supported by the grants from Ministry of Education, Science, Sports and Culture of Japan. K. N. was supported by research fellowships from the Japan Society for the Promotion of Science (JSPS) for young scientists.

\section{REFERENCES}

1. Gibbs, E. P. J. and Rweyemamu, M. M. 1977. Vet Bull. (London) 47: 317-343.

2. Montgomery, R. I., Warner, M. S., Lum, B. and Spear, P. G. 1996. Cell 87: 427-436.

3. Morahan, P. S., Mama, S., Anaraki, F. and Leary, K. 1989. J. Virol. 63: 2300-2307.

4. Murata, T., Takashima, Y., Xuan, X. and Otsuka, H. 1999. Virus Res. 61: 29-41.

5. Nakamichi, K. and Otsuka, H. 2000. Intervirology 43: 87-94.

6. Nakamichi, K., Ohara, K., Kuroki, D. and Otsuka, H. 2000. Virus Res. 68: 175-181.

7. Nakamichi K., Ohara, K., Matsumoto, Y. and Otsuka, H. 2000. J. Vet. Med. Sci. 62: 965-970.

8. Nixdorf, R., Schmidt, J., Karger, A. and Mettenleiter T. C. 1999. J. Virol. 73: 8019-8026.

9. Perez, A. and Fuller, A. O. 1998. Virus Res. 58: 21-34.

10. Sawitzky, D., Hampl, H. and Habermehl, K. O. 1990. Arch. Virol. 115: 309-316. 


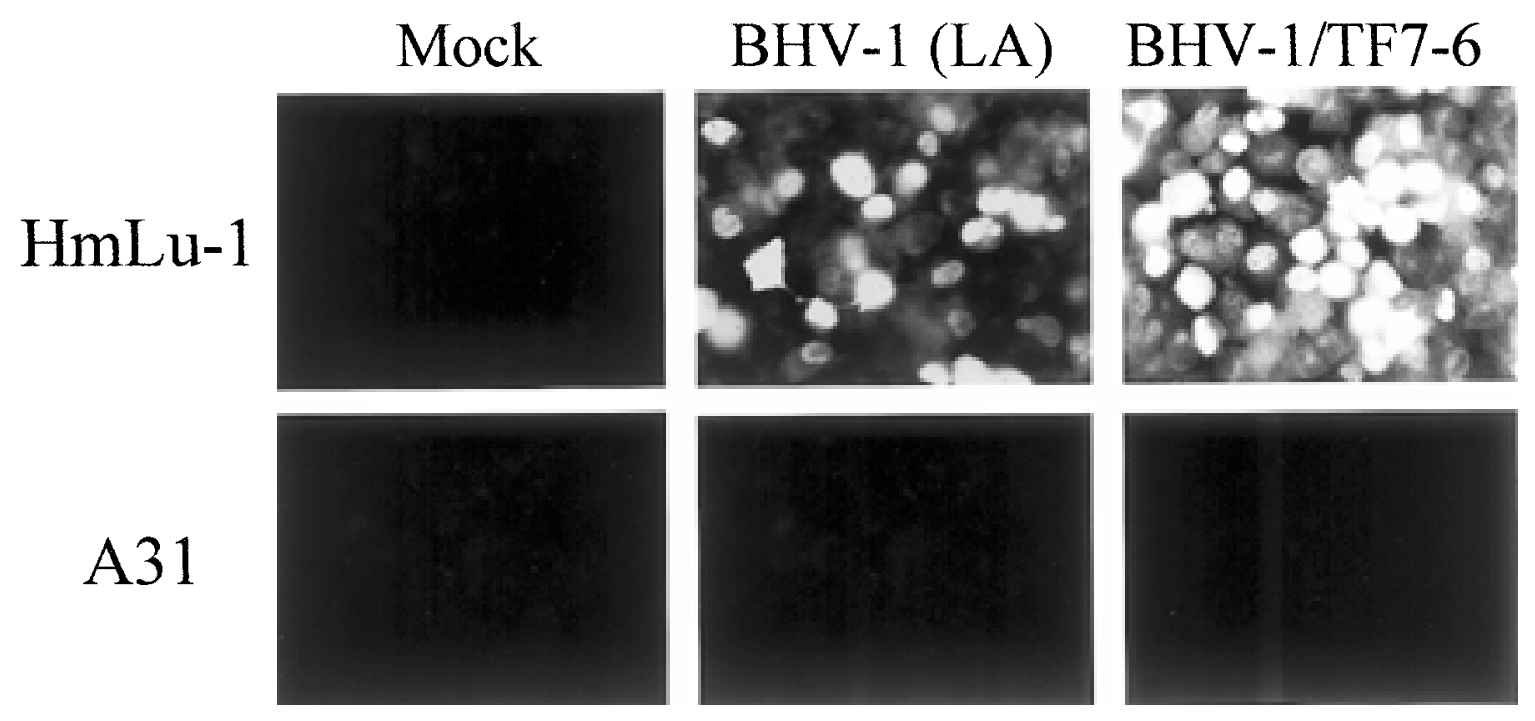

Fig. 3. Immunofluorescence assay of BICP4 expression. HmLu-1 (upper panels) and A31 cells (lower panels) were mock infected or infected with BHV-1 (LA) and BHV-1/TF7-6 at an MOI of 10. At $24 \mathrm{hr}$ p.i., cells were fixed, reacted with anti-BICP4 mouse antiserum and stained with FITC-conjugated anti-mouse $\operatorname{IgG}$ antibody. Original magnification, $\times 400$.

11. Schwyzer, M., Vlcek, C., Menekse, O., Fraefel C. and Paces, V. 1993. Virology 197: 349-357.

12. Subramanian, G., McClain, D. S., Perez, A. and Fuller, A. O. 1994. J. Virol. 68: 5667-5676.

13. Subramanian, G., LeBlanc, R. A., Wardley, R. C. and Fuller, A. O. 1995. J. Gen. Virol. 76: 2375-2379.
14. Tikoo, S. K., Campos, M. and Babiuk, L. A. 1995. Adv. Virus Res. 45: 191-223.

15. Walter, I. and Nowotny, N. 1999. Arch. Virol. 144: 1827-2836.

16. Wang, K., Hirayasu, K., Ishizawa, M. and Kobayashi, Y. 1994. Nucleic Acids Res. 22: 1774-1775. 